\title{
Improved reduced representation bisulfite sequencing for epigenomic profiling of clinical samples
}

\author{
Yew Kok Lee ${ }^{1 \dagger}$, Shengnan Jin ${ }^{1 \dagger}$, Shiwei Duan ${ }^{1}$, Yen Ching Lim, Desmond PY Ng${ }^{1}$, Xueqin Michelle Lin ${ }^{1}$, \\ George $\mathrm{SH}$ Yeo $^{2}$ and Chunming Ding ${ }^{1 *}$
}

\begin{abstract}
Background: DNA methylation plays crucial roles in epigenetic gene regulation in normal development and disease pathogenesis. Efficient and accurate quantification of DNA methylation at single base resolution can greatly advance the knowledge of disease mechanisms and be used to identify potential biomarkers. We developed an improved pipeline based on reduced representation bisulfite sequencing (RRBS) for cost-effective genome-wide quantification of DNA methylation at single base resolution. A selection of two restriction enzymes ( $\mathrm{Taq}^{\mathrm{a}} \mathrm{I}$ and $\mathrm{Mspl}$ ) enables a more unbiased coverage of genomic regions of different $\mathrm{CpG}$ densities. We further developed a highly automated software package to analyze bisulfite sequencing results from the Solexa GAllx system.

Results: With two sequencing lanes, we were able to quantify $~ 1.8$ million individual CpG sites at a minimum sequencing depth of 10 . Overall, about $76.7 \%$ of CpG islands, 54.9\% of CpG island shores and $52.2 \%$ of core promoters in the human genome were covered with at least $3 \mathrm{CpG}$ sites per region.

Conclusions: With this new pipeline, it is now possible to perform whole-genome DNA methylation analysis at single base resolution for a large number of samples for understanding how DNA methylation and its changes are involved in development, differentiation, and disease pathogenesis.
\end{abstract}

Keywords: DNA methylation, Epigenetics, Bisulfite sequencing, Clinical sequencing

\section{Background}

Epigenetics is the study of heritable changes in gene expression without altering the DNA sequence. DNA methylation, by the addition of a methyl group to the carbon-5 position of the cytosine residues, is one important epigenetic regulatory mechanism [1]. In mammalian development, genome-wide de novo methylation occurs during germ cell development and implantation, while genome-wide demethylation occurs during primordial germ cell development and shortly after fertilization [2]. DNA methylation and demethylation also occur during cell differentiation and reprogramming [3]. Abnormal DNA methylation levels, either hypermethylation or hypomethylation in specific genes, are

\footnotetext{
* Correspondence: cmding@gmail.com

†Equal contributors

'Singapore Institute for Clinical Sciences, Agency for Science, Technology and Research (A*STAR), 117609 Singapore, Singapore

Full list of author information is available at the end of the article
}

also frequently observed in pathologic states, particularly in cancer [4].

Accurate quantification of DNA methylation is essential to decipher mechanisms and pathways regulated epigenetically in development and pathogenesis. Many techniques have been developed for the detection and quantification of DNA methylation [reviewed by $[5,6]]$.

The combination of bisulfite conversion and highthroughput sequencing (Bis-Seq) offers the most quantitative method for DNA methylation analysis at single base resolution. Unmethylated cytosine is converted to uracil by sodium bisulfite treatment while methylated cytosine remains unchanged [7]. Genomic DNA after bisulfite conversion is amplified by PCR and then sequenced at high depth, yielding quantitative measurements of individual cytosine methylation.

While genome-wide Bis-Seq was achieved, published studies typically only analyzed a few samples from cultured 
cell lines [8-10]. When it is required to analyze tens or hundreds of clinical samples, reduced representation bisulfite sequencing (RRBS) may be the method of choice [11]. The RRBS method makes use of restriction enzyme digestion to selectively analyze genomic regions enriched for $\mathrm{CpG}$ sites in a methylation-independent manner, thus achieving a high coverage of $\mathrm{CPG}$ rich regions while greatly reducing sequencing read requirement. The current RRBS protocol uses one single enzyme MspI targeting 5'-CCGG-3' for DNA fragment selection, which results in selectively covering CpG rich regions [12]. However, coverage for non $\mathrm{CpG}$ rich regions is generally poor. Recent epigenomic data suggest that $\mathrm{CpG}$ poor regions distal to core promoters perform important regulatory functions [8]. Thus, an improvement over the current RRBS protocol to cover CpG poor regions is essential.

In this paper, we performed a comprehensive in silico analysis of restriction enzyme digestion of the human genome. A combination of two enzymes, $\mathrm{Taq}^{\alpha} \mathrm{I}$ and MspI, yielded the most desirable coverage of the $\mathrm{CpG}$ sites in both $\mathrm{CpG}$ rich and $\mathrm{CpG}$ not-so-rich regions. We also describe how Bis-Seq data are analyzed. We believe a detailed description of the entire improved RRBS pipeline would greatly facilitate epigenomic studies requiring the analysis of large numbers of samples.

\section{Results}

We made key improvements in genome-wide DNA methylation analysis by RRBS, in both the experiment steps and the data analysis pipeline. Firstly, through extensive in silico analysis we chose to digest the human genomic DNA with a combination of two enzymes, MspI and $\mathrm{Taq}^{\alpha} \mathrm{I}$, which allowed us to cover both $\mathrm{CpG}$ islands (CGIs) as well as genomic regions outside CGIs. Secondly, we removed a size range between 198 to $206 \mathrm{bp}$ (with the adaptor) that are repetitive sequence rich. Lastly, we developed a highly automated bioinformatics pipeline with a detailed step-by-step explanation of both quality control and data analysis.

We used the above pipeline for the analysis of a large number of clinical samples such as human placenta, umbilical cord and leukocytes. As an example, we provide data generated from one human buffy coat DNA.

\section{Selection of restriction enzymes and DNA fragment size range}

We systematically analyzed 289 motifs recognized by restriction enzymes with in silico digestion of the human genome (GRCh37/hg19, Feb. 2009 Assembly). Further analyses were carried out with the following considerations: 1) CpG methylation sensitivity and commercial availability of the restriction enzymes; 2 ) genome-wide coverage of the different genomic regions [promoters (defined as -1000 bp to +500 relative to a transcription start site), CGIs, CpG island shores (CGSs), gene bodies, transcription termination regions (TTRs, defined as $-500 \mathrm{bp}$ to $+500 \mathrm{bp}$ relative to a transcription termination site)] in the fragments generated by enzyme digestions; 3) size distribution of the fragments; and 4) single enzyme digestion and double enzyme digestions. In the end, MspI and $\mathrm{Taq}^{\alpha} \mathrm{I}$ double enzyme digestion was chosen for the RRBS protocol. A total of 3,810,058 fragments from the human genome are generated by double digestion of the two enzymes, among which 450,689 fragments are between 80 to $160 \mathrm{bp}$. Within the range of 80 to $160 \mathrm{bp}$, there is an enrichment of repetitive sequences between 128 to $136 \mathrm{bp}$, as predicted by in silico digestion and validated by a clear DNA band at this size range by gel electrophoresis (around $200 \mathrm{bp}$ with the adaptors) (Figure 1). We removed this DNA band in the lab protocol.

We compared the in silico digested DNA fragments (80-160 bp) by MspI and $\mathrm{Taq}^{\alpha} \mathrm{I}$ double digestion and MspI single digestion (single digestion is from the original RRBS protocol). With double digestion, we observed (Table 1) substantial improvements in CpGs in non-CGI regions (41.8\% increase), as well as modest improvements in total CpGs in CGIs (7.4\% increase), CGIs (6.3\% increase) and promoters (12.7\% increase).

\section{Quality control for sequencing results}

Our pipeline assesses DNA library and sequencing result quality in a number of different aspects.

\section{Quality of sequencing reads}

Each flow cell used for DNA sequencing contains eight lanes. Each lane contains 120 tiles. We quantified the number of total reads and the number of good reads (passing a cut-off score of 30) for each tile (Additional file 1: Figure S1). Typically, percentages of good reads were around $70-90 \%$. Additionally, we assessed the percentage of alignment for each library. A typical alignment percentage defined as number of uniquely aligned reads over total pass filtered reads was around 55-65\%.

\section{Library quality}

The preparation protocol for sequencing library by RRBS results in a number of predictable features. Firstly, the first three nucleotides for read 1 of paired-end sequencing should be either CGG/TGG (MspI), or CGA/TGA ( $\left.\mathrm{Taq}^{\alpha} \mathrm{I}\right)$ for the enzymatically generated fragments, while those for read 2 should be CAA. Indeed, these predicted sequencing ends were dominant (97.8\% for CGG/TGG/ CGA/TGA in read 1 and $91.9 \%$ for CAA in read 2) (Additional file 1: Table S1). Secondly, read 1 should map to the positive strand of C2T reference genome, or negative strand of G2A reference genome, while read 2 should be the exact opposite to read 1 . This was also confirmed after sequencing alignment (Additional file 1: 


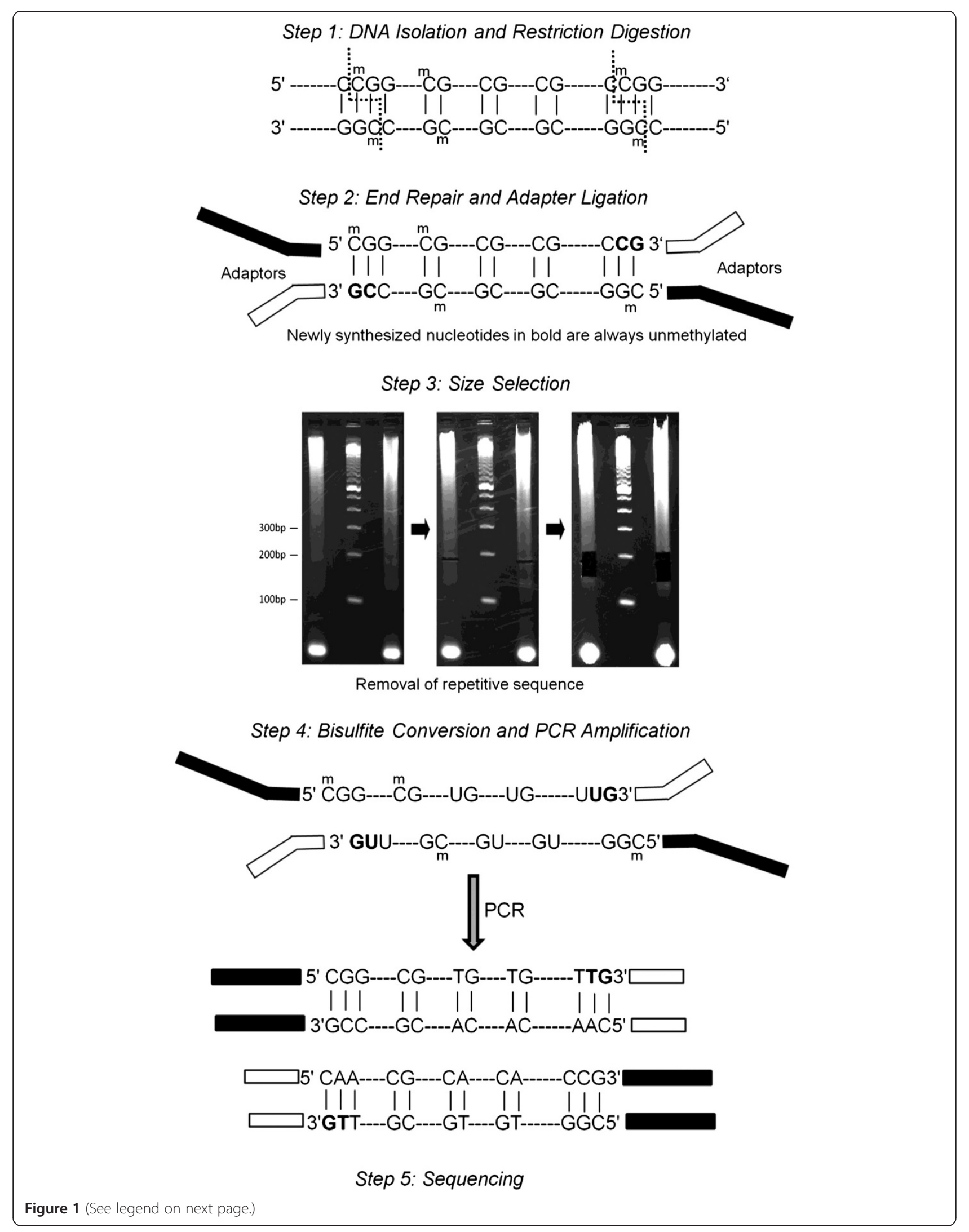


(See figure on previous page.)

Figure 1 Key laboratory steps in RRBS. The isolated DNA from the samples are digested by two restriction enzymes (Taq I and Mspl). The fragments are then end-repaired and ligated with adapters. The ligated DNA are size-selected and repetitive sequences are removed. PCR amplification is performed after bisulfite conversion. Illumina GAllx system is used for high-throughput sequencing. Newly added bases (marked in bold in step 2) are always unmethylated and thus discarded in the downstream analysis for CpG methylation.

Table S2). Thirdly, the relative ratio of cytosine over guanine in each read follows a specific pattern. The $\mathrm{C} / \mathrm{G}$ ratio should be $<1$ in read 1 and $>1$ in read 2 , as was seen in the sequencing reads (Additional file 1: Table S3). Fourthly, the size distribution of library inserts was analyzed. As expected, the fragment size ranged from 80 to $160 \mathrm{bp}$, with low abundance shown around $130 \mathrm{bp}$ since we removed the repetitive sequences around this size range (Additional file 1: Figure S2).

\section{Bisulfite conversion rate}

We estimated the conversion rate using cytosines in nonCpG regions, assuming any unconverted cytosine was due to incomplete conversion. This is likely to over-estimate the non-conversion rate, particularly in samples with cytosine methylation in non-CpG sites, such as in embryonic stem cells [10].

\section{Removal of CpGs with potential polymorphisms}

As polymorphisms within known CpG sites are likely to cause wrong interpretation of DNA methylation, we implemented a filtering step. A CpG site was discarded if a probable polymorphism was identified. As an example, in one sample we removed 25,135 CpGs out of 1,282,265 CpGs with a minimum sequencing depth of 10 .

\section{Coverage cutoff}

We used a minimum sequencing depth of 10 as the cutoff for inclusion for DNA methylation analysis. However, it is possible that a lower cutoff may be sufficient for many downstream analyses, as suggested by a comparison between RRBS and Illumina Infinium $450 \mathrm{~K}$ array [13]. The numbers of CpG sites at different depth were shown in Figure 2A.

\section{Coverage of $\mathrm{CpGs}$ in different genomic regions}

Approximately 1.8 million CpG sites with minimum sequencing depth of 10 were included in the analyses.
As shown in Figure 2B, 41\% of the CpG sites were located within the CGIs, $13 \%$ of them were located in CGSs and the remaining CpG sites (46\%) were located in CpG poor regions outside of CGIs or CGSs.

As shown in Figure 2C, 18\% of the CpG sites were located in the promoter regions overlapping with CGIs while $4 \%$ of the CpG sites were located in the promoter regions not overlapping with CGIs. Additionally, 39\% of the $\mathrm{CpG}$ sites were in intragenic regions, $37 \%$ of them were in the intergenic regions and the remaining $2 \%$ were in the TTR regions.

Genomic regions with at least three CpGs covered at a minimum sequencing depth of 10 were considered to be covered. With 2 lanes of Solexa sequencing, we achieved $52.2 \%$ coverage for core promoters, $76.7 \%$ coverage for CGIs and $54.9 \%$ coverage for CGSs (Table 2).

As shown in Figure 2D, 13\% of the covered regions overlapped with CGIs, $17 \%$ of them overlapped with CGSs and the remaining regions $(70 \%)$ were located outside of CGIs or CGSs.

As shown in Figure 2E, 62\% of covered regions were in the intergenic regions. Only $6 \%$ of the covered regions were in the promoters overlapping with CGIs and 3\% were in the promoters not overlapping with any CGI. Twenty eight percent of the regions were found in the intragenic regions and the remaining $2 \%$ were in the TTR regions.

\section{Discussion}

Epigenetics changes such as chromatin assembly, histone modifications and DNA methylations are mediators between gene and environment by regulating genomic structures and gene transcription. Genome-wide DNA methylation profiling of human cohorts (or epigenome wide association studies, EWASs) may complement genetic association studies in identifying genes involved in complex diseases. A number of platforms are available for "genomewide” DNA methylome analysis. True genome-wide DNA

Table 1 Comparison of in silico digestions (double vs. single digestion)

\begin{tabular}{|c|c|c|c|}
\hline & $\begin{array}{l}\text { Covered by Mspl } \\
\text { single digestion }\end{array}$ & $\begin{array}{l}\text { Covered by Mspl and } \\
\operatorname{Taq}^{\alpha} \mathrm{I} \text { double digestion }\end{array}$ & $\begin{array}{l}\text { Percentage of increase } \\
\text { (double vs. single digestion) }\end{array}$ \\
\hline CpGs in CGI regions & $1,098,462$ & $1,180,058$ & $7.4 \%$ \\
\hline CpGs in non-CGl regions & $1,919,174$ & $2,720,858$ & $41.8 \%$ \\
\hline $\mathrm{CGIS}^{*}$ & 20,227 & 21,511 & $6.3 \%$ \\
\hline Promoters* ${ }^{*}(-1000$ to +500 bp) & 24,520 & 27,633 & $12.7 \%$ \\
\hline
\end{tabular}

*by at least $3 \mathrm{CpGs}$ covered in the genomic region. 


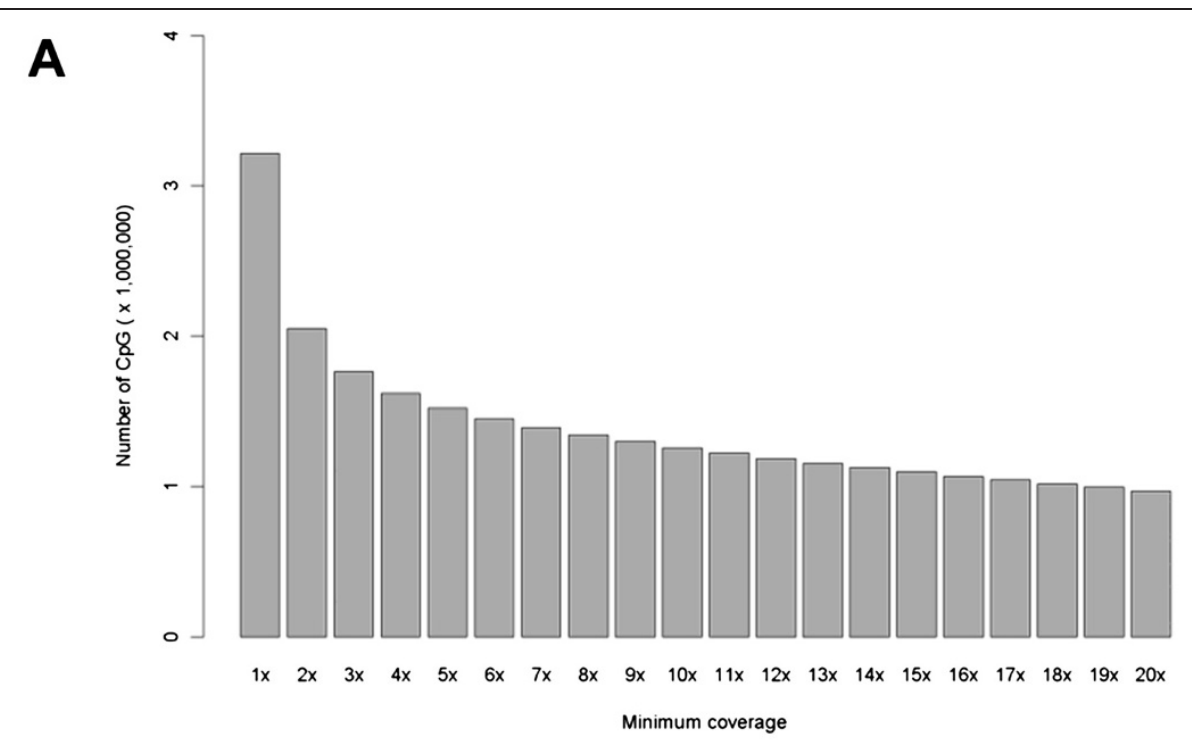

B

Distribution of CpGs in CGIs/CGSs/Others

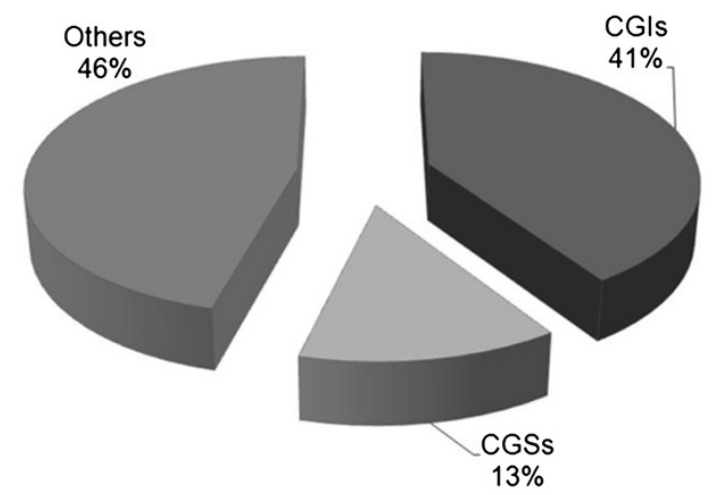

C

\section{Distribution of $\mathrm{CpGs}$ in}

Promoter/TTR/Intragenic/Intergenic Regions

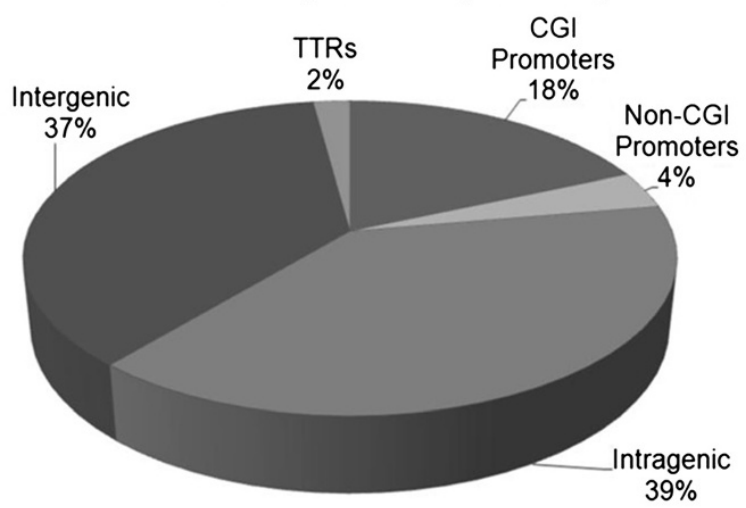

D

\section{Distribution of Covered CGIs/CGSs/Others}

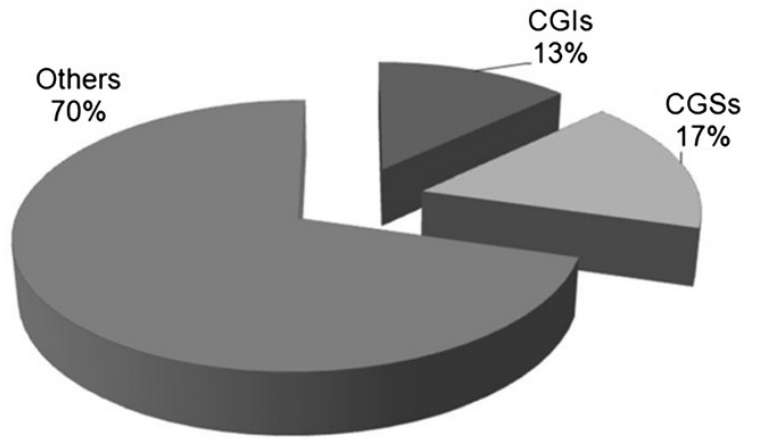

E

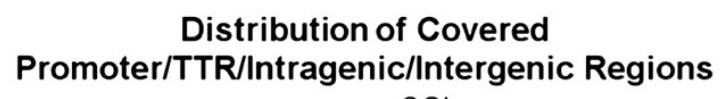
CGI

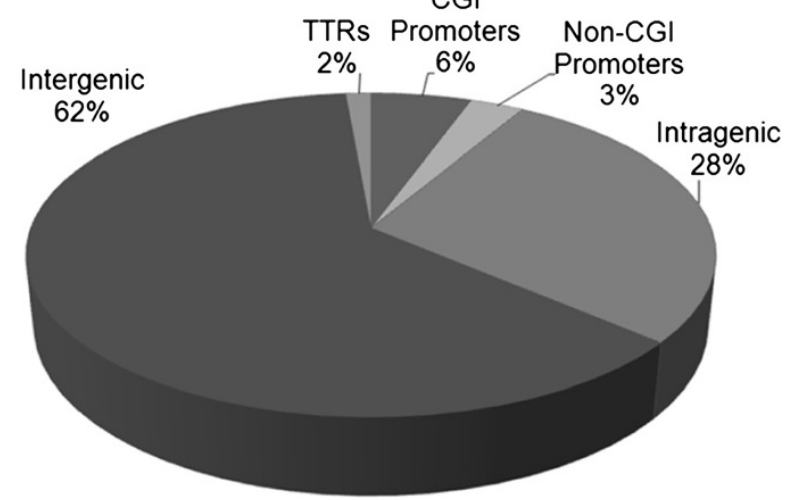

Figure $\mathbf{2}$ (See legend on next page.) 
(See figure on previous page.)

Figure $\mathbf{2}$ Coverage of CpGs and genomic regions by RRBS. (A) An example for number of CpG sites with different minimum sequencing depths; (B) Distribution of CpGs in CGIs/CGSs/Others, using a sequencing depth $\geq 10$ as the cutoff; (C) Distribution of CpGs in Promoter/TTR/ Intragenic/Intergenic regions; (D) Distribution of genomic regions in CGIs/CGSs/others. (E) Distribution of genomic regions in promoter/TTR/ Intragenic/Intergenic regions. A genomic region was considered covered if at least three CpGs within the region were sequenced at a depth $\geq 10$.

methylome analysis by bisulfite sequencing represents the most comprehensive coverage, yet it is cost prohibitive for analyzing a reasonable number of samples. Two platforms, Illumina $450 \mathrm{~K}$ Infinium Array and RRBS, appear to be the immediate choices for EWASs [14]. One potential problem with the $450 \mathrm{~K}$ Array and the current RRBS approach is poor coverage for $\mathrm{CpG}$ poor regions, which may miss important regulatory regions [8].

Through extensive in silico enzyme digestion of the human genome, we selected a combination of two enzymes $\left(\mathrm{Taq}^{\alpha} \mathrm{I}\right.$ and $\mathrm{MspI}$ ) for a more unbiased coverage of the human genome. We further removed a DNA fragment size range enriched for repetitive sequences so that more reads are usable for alignment. We provide here a comprehensive description for the entire process of the improved RRBS pipeline so that it can be easily adopted by researchers. We also extensively compared the RRBS and $450 \mathrm{~K}$ Array platforms by analyzing seven umbilical cord DNA samples with both methods [13]. The two methods are highly complementary as they cover mostly different CpGs and genomic regions. Thus, it is possible to combine the two methods for a more complete coverage for EWASs.

\section{Conclusions}

With this new pipeline, it is now possible to perform whole-genome DNA methylation analysis at single base resolution for a large number of samples for understanding how DNA methylation and its changes are involved in development, differentiation, and disease pathogenesis.

\section{Methods}

\section{Experimental protocol}

A schematic view for the key steps in the lab protocol is outlined in Figure 1.

\section{DNA isolation and restriction enzyme digestion}

One to five microgram of high molecular weight (>10 kb) genomic DNA was used for each library preparation. Each
DNA sample was subjected to sequential restriction enzyme digestion with MspI and $\mathrm{Taq}^{\alpha} \mathrm{I}$ (New England Biolabs). Briefly, DNA was first incubated with $150 \mathrm{U}$ of MspI in a $80 \mu \mathrm{L}$ system containing $1 \times$ Buffer 4 for $2 \mathrm{hrs}$ at $37^{\circ} \mathrm{C}$, and the enzyme was inactivated by heating at $80^{\circ} \mathrm{C}$ for $20 \mathrm{~min}$. Additional $20 \mu \mathrm{L}$ solution containing $150 \mathrm{U}$ of $\mathrm{Taq}^{\alpha} \mathrm{I}$, $1 \times$ Buffer 4 and $1 \mu \mathrm{L}$ of $100 \times$ BSA was added to the same reaction tube, and the DNA sample was further incubated at $65^{\circ} \mathrm{C}$ for $2 \mathrm{hrs}$. The second enzyme was then inactivated by heating at $80^{\circ} \mathrm{C}$ for $20 \mathrm{~min}$. The doubledigested product was purified with the QIAquick PCR Purification Kit (QIAGEN GmbH, Germany), and all was used for library preparation.

\section{End repair and adapter ligation}

The DNA fragments with 5'-CG-3' overhangs generated by the restriction enzyme digestion were end-repaired, 3 '-end-adenylated, and adapter-ligated using the ChIPSeq Sample Preparation Kit (Illumina). Illumina's RRBS for Methylation Analysis protocol was followed, except that $10 \mu \mathrm{L}$ of Early Access Methylation Adapter Oligo (Illumina) was used and the ligation was performed for $15 \mathrm{~min}$ at $20^{\circ} \mathrm{C}$ in the adapter-ligation step.

\section{Size selection of adapter-ligated fragments}

Two different sizes of fragments (150-197 bp and 207-230 bp) were selected by gel electrophoresis with $3 \%$ agarose gel, corresponding to DNA fragments of 80 to $160 \mathrm{bp}$ without the adapter. DNA fragments with high abundance of repetitive sequences (between 198 to $206 \mathrm{bp}$ with the adaptor, based on in silico analysis) were removed using GeneCatcher Gel Excision Kit (Gel Company), and the remaining fragments between 150 to 230 bp were purified by MinElute Gel Extraction Kit (QIAGEN GmbH, Germany).

\section{Bisulfite conversion and amplification}

The purified fragments were then subject to bisulfite conversion using the EZ DNA Methylation-Gold Kit

Table 2 Genome-wide coverage by RRBS

\begin{tabular}{llll}
\hline & Total in human genome & Covered by RRBS & Percentage \\
\hline CpGs & $56,434,896$ & $1,837,502$ & $3.3 \%$ \\
CGls & 27,718 & 21,252 & $76.7 \%$ \\
CGSs ( \pm 2 KB from CGl) & 49,300 & 27,074 & $54.9 \%$ \\
Promoters (-1000 to +500 bp) & 44,399 & 23,168 & $52.2 \%$ \\
\hline
\end{tabular}


(Zymo Research), according to manufacturer's instructions. The converted DNA was PCR amplified with $1 \mathrm{x}$ reaction buffer, an additional $1.5 \mathrm{mM}$ of $\mathrm{MgCl}_{2}, 300 \mu \mathrm{M}$ of dNTP mix, $500 \mathrm{nM}$ each of PCR primer PE 1.0 and 2.0, and $2.5 \mathrm{U}$ of HotStarTaq DNA polymerase (QIAGEN). The thermocycling condition was $15 \mathrm{~min}$ at $94^{\circ} \mathrm{C}$ for heat activation, and 8-12 cycles of $20 \mathrm{sec}$ at $94^{\circ} \mathrm{C}, 30 \mathrm{sec}$ at $65^{\circ} \mathrm{C}$ and $30 \mathrm{sec}$ at $72^{\circ} \mathrm{C}$, followed by a 5 -min final extension at $72^{\circ} \mathrm{C}$. The PCR products were purified by gel electrophoresis.

\section{Quantification and quality check of DNA libraries and sequencing}

The DNA libraries were quantified using Agilent 2100 Bioanalyzer (Agilent Technologies). Typically final libraries contain 200 fmoles of fragments. Paired-end sequencing $(2 \times 36 \mathrm{bp})$ was performed on the Illumina Genome Analyzer IIx platform, as per manufacturer's instructions.

\section{Data analysis pipeline}

\section{Reference genome conversion}

The human reference genome was converted into two reference genomes corresponding to the forward strand (Watson Strand) and the reverse strand (Crick Strand). The C2T converted reference genome was derived by converting all cytosines to thymines. The G2A converted reference genome was derived by converting all guanines to adenosines.

\section{Initial quality control of sequencing reads}

The paired-end 36 bp reads were filtered based on their Phred scores [15], using a cutoff of 30 [16] which indicates a base calling error of 0.001 .

The quality of reads was examined by plotting the number of total reads and the numbers of reads passing the quality cut-off score from each of the 120 tiles of each sequencing lane (Additional file 1: Figure S1).

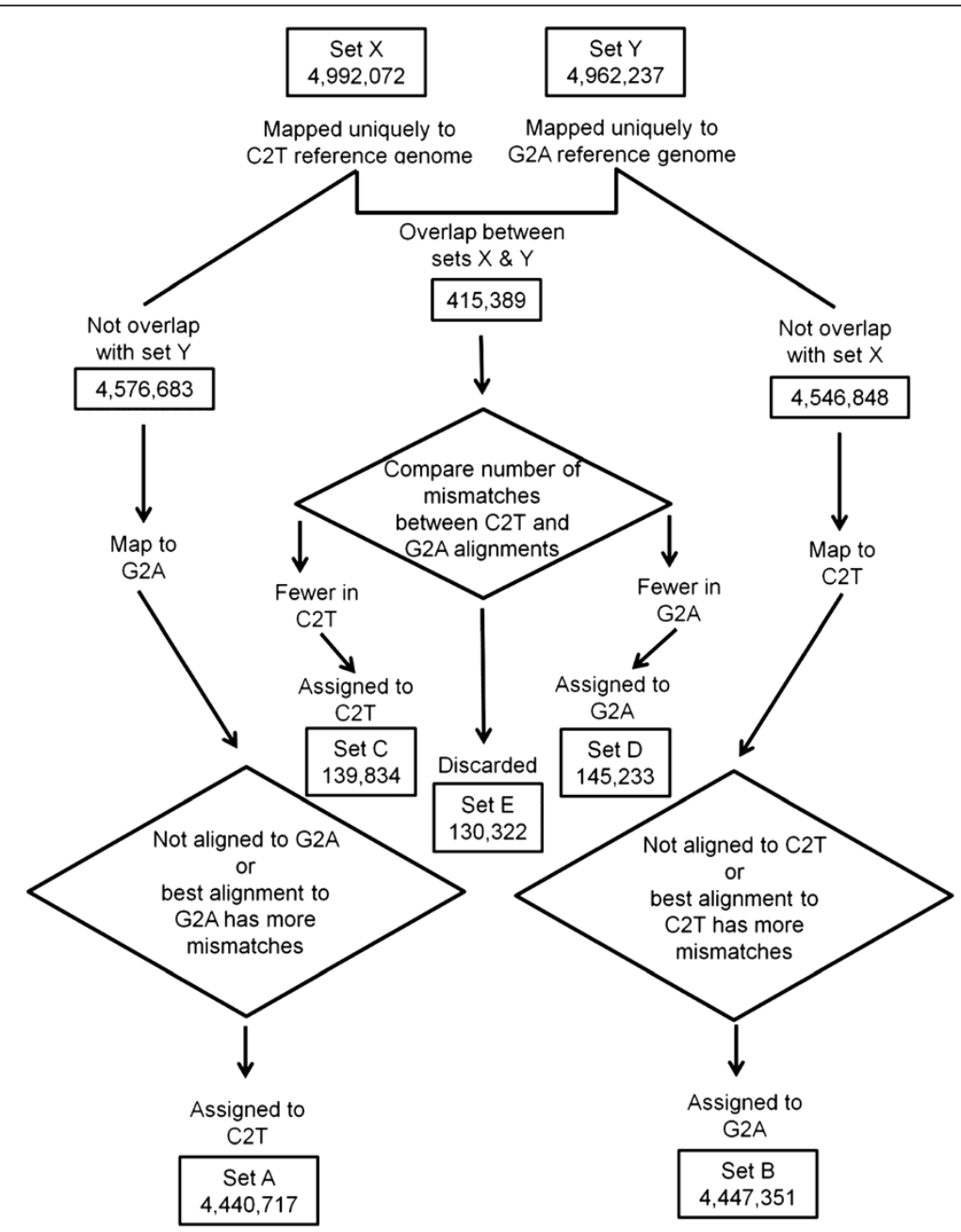

Figure 3 Sequencing reads alignment to two reference genomes, C2T and G2A reference genomes. Due to the pointer size limitation by the Bowtie program, reads alignment needs to be performed using the two reference genomes separately. Cross check and comparison are made subsequently to remove reads not uniquely aligned. At the end, sets $A$ and $C$ are read pairs aligned to C2T reference genome, while sets $B$ and $\mathrm{D}$ are read pairs aligned to the G2A reference genome. 
Additionally, enzymatically generated DNA fragments should end with specific tri-nucleotides such as CGG/ TGG/CGA/TGA/CAA while DNA fragments from random shearing may end with any sequence. Thus sequencing library quality was also assessed by calculating the percentages of reads ending by expected tri-nucleotides.

\section{Reads conversions}

The design of the assays predicts that read 1 sequence should have a $C / G$ ratio $<1$ while read 2 sequence should have a $\mathrm{C} / \mathrm{G}$ ratio $>1$, if bisulfite conversion is complete and there is no non-CpG cytosine methylation. Thus, only paired-end reads that followed the above rules were used.

\section{Sequence alignment}

Bowtie, an ultrafast, memory-efficient short read aligner was used for aligning the sequencing reads [17]. Unfortunately, Bowtie cannot hold a combined index for both $\mathrm{C} 2 \mathrm{~T}$ and G2A reference genomes due to the fact that it uses a 32-bit pointer and thus it can handle up to a theoretical maximum of $2^{32}-1$ (slightly more than 4 billions). Thus, two separate Bowtie indexes were created for the $\mathrm{C} 2 \mathrm{~T}$ and G2A reference genomes, respectively. We thus developed a process for sequencing reads alignment (Figure 3).

All pass-filtered, converted reads were aligned to the $\mathrm{C} 2 \mathrm{~T}$ and G2A reference genomes separately, using paired-end reads alignments. Reads set X (4,994,072 pairs) were those read pairs that aligned uniquely to the $\mathrm{C} 2 \mathrm{~T}$ reference genome, while set $Y(4,962,237)$ were those that aligned uniquely to the G2A reference genome. Overlapping read pairs between set $X$ and set $Y(415,389)$ were then compared between the $\mathrm{C} 2 \mathrm{~T}$ and G2A alignments with regards to the number of mismatched bases, resulting in further separation of sets C $(139.834), D(145,233)$ and $E(130,322)$. The read pairs in set $X$ not overlapping with set $\mathrm{Y}$ were then aligned to the G2A reference genome for further comparison, resulting in set A $(4,440,717)$. Similarly, set $B$ was obtained.

At the end, sets $A$ and $C$ were read pairs aligned to $C 2 T$ reference genome, while sets $\mathrm{B}$ and $\mathrm{D}$ were read pairs aligned to the G2A reference genome. All other read pairs were discarded since they were not able to align uniquely to the combined C2T and G2A reference genomes.

\section{Excluding newly filled-in Cytosines}

The newly added cytosines in the "End Repair" step are always unmethylated, regardless of their original methylation status. Such cytosines were excluded in our analysis (Figure 1).

\section{Removing of CpGs overlapping with potential polymorphisms}

Polymorphisms overlapping with CpGs may introduce abnormalities in the data. In this regard, CpG sites with percentage of dinucleotide ' $\mathrm{XY}$ ' other than ' $\mathrm{CG}$ ' or ' $\mathrm{TG}$ ' greater than $20 \%$ were deemed to be probably polymorphic for the sample and were excluded for further analysis.

\section{Calculating each CPG sequencing depth and methylation level}

Methylation level of each $\mathrm{CpG}$ site was calculated as below:

Methylation level for a $\mathrm{CpG}=$ Count of Cytosine/ (Count of Cytosine + Count of Thymine)*100\%.

Cytosines in non-CpG positions were used to calculated bisulfite conversion rate, assuming any unconverted cytosine was due to incomplete conversion.

The pipeline is available for academic, non-commercial use upon request.

\section{Additional file}

Additional file 1: Figure S1. Assessment of Reads Quality for Each Sequencing Run. Good: read pairs passing the Phred score cutoff of 30. Total: total raw read pairs. Figure S2. Distribution of Library Insert Length. Table S1. Counts of the first three nucleotides in the sequencing reads. For read 1, the first three nucleotides were expected to be CGG/TGG/ CGATTGA. For read 2, the first three nucleotides were expected to be CAA. Table S2. Number of reads aligned to the positive or negative strand of the two converted reference genomes. C2TRef: C2T reference genome; G2ARef: G2A reference genome. Table S3. C/G ratios in the sequencing reads. In the first three cases (row 2 to 4 ), the read pairs showed expected $\mathrm{C} / \mathrm{G}$ ratios and were thus used for alignment. All others (row 5) were excluded for further analysis.

\section{Abbreviations}

RRBS: Reduced representation bisulfite sequencing; Bis-Seq: Bisulfite conversion and high-throughput sequencing; CGls: CpG islands; CGSs: CpG island shores; TTRs: Transcription termination regions; EWASs: Epigenome wide association studies.

\section{Competing interests}

The authors declare that they have no competing interests.

\section{Authors' contributions}

YKL, SD and YCL performed the computational analysis. SJ, DPYN and XML performed the experiments. GSHY provided the samples. YKL, SJ and CD wrote the paper. All authors read and approved the final manuscript.

\section{Acknowledgements}

C.D. and G.Y. are supported by a Bench to Bedside grant (09/1/50/19/622) from BMRC-NMRC

\section{Author details}

${ }^{1}$ Singapore Institute for Clinical Sciences, Agency for Science, Technology and Research (A*STAR), 117609 Singapore, Singapore. 'KK Women's and Children's Hospital, 229899 Singapore, Singapore.

\section{Received: 11 October 2013 Accepted: 4 January 2014}

Published: 9 January 2014

\section{References}

1. Nakao M: Epigenetics: interaction of DNA methylation and chromatin. Gene 2001, 278(1-2):25-31.

2. Reik W, Dean W, Walter J: Epigenetic reprogramming in mammalian development. Science 2001, 293(5532):1089-1093.

3. Chen ZX, Riggs AD: DNA methylation and demethylation in mammals. J Biol Chem 2011, 286(21):18347-18353.

4. Robertson KD: DNA methylation and human disease. Nat Rev Genet 2005, 6(8):597-610. 
5. Bock C, Tomazou EM, Brinkman AB, Muller F, Simmer F, Gu H, Jager N, Gnirke A, Stunnenberg HG, Meissner A: Quantitative comparison of genome-wide DNA methylation mapping technologies. Nat Biotechnol 2010, 28(10):1106-1114.

6. Laird PW: Principles and challenges of genomewide DNA methylation analysis. Nat Rev Genet 2010, 11(3):191-203.

7. Frommer M, McDonald LE, Millar DS, Collis CM, Watt F, Grigg GW, Molloy $\mathrm{PL}$, Paul CL: A genomic sequencing protocol that yields a positive display of 5-methylcytosine residues in individual DNA strands. Proc Natl Acad Sci USA 1992, 89(5):1827-1831.

8. Stadler MB, Murr R, Burger L, Ivanek R, Lienert F, Scholer A, van Nimwegen E, Wirbelauer C, Oakeley EJ, Gaidatzis D, et al: DNA-binding factors shape the mouse methylome at distal regulatory regions. Nature 2011, 480(7378):490-495.

9. Laurent L, Wong E, Li G, Huynh T, Tsirigos A, Ong CT, Low HM, Kin Sung KW, Rigoutsos I, Loring J, et al: Dynamic changes in the human methylome during differentiation. Genome Res 2010, 20(3):320-331.

10. Lister R, Pelizzola M, Dowen RH, Hawkins RD, Hon G, Tonti-Filippini J, Nery $J R$, Lee L, Ye Z, Ngo QM, et al: Human DNA methylomes at base resolution show widespread epigenomic differences. Nature 2009, 462(7271):315-322.

11. Meissner A, Gnirke A, Bell GW, Ramsahoye B, Lander ES, Jaenisch R: Reduced representation bisulfite sequencing for comparative high-resolution DNA methylation analysis. Nucleic Acids Res 2005, 33(18):5868-5877.

12. Gu H, Bock C, Mikkelsen TS, Jager N, Smith ZD, Tomazou E, Gnirke A, Lander ES, Meissner A: Genome-scale DNA methylation mapping of clinical samples at single-nucleotide resolution. Nat Methods 2010, 7(2):133-136.

13. Pan H, Chen L, Dogra S, Teh AL, Tan JH, Lim Yl, Lim YC, Jin S, Lee YK, Ng PY, et al: Measuring the methylome in clinical samples: improved processing of the Infinium Human Methylation450 BeadChip Array. Epigenetics 2012, 7(10):1173-1187.

14. Rakyan VK, Down TA, Balding DJ, Beck S: Epigenome-wide association studies for common human diseases. Nat Rev Genet 2011, 12(8):529-541.

15. Ewing B, Green P: Base-calling of automated sequencer traces using phred. II. Error probabilities. Genome Res 1998, 8(3):186-194.

16. illumina: Quality Scores for Next-Generation Sequencing. Technical Note: Sequencing 2011.

17. Langmead B, Trapnell C, Pop M, Salzberg SL: Ultrafast and memory-efficient alignment of short DNA sequences to the human genome. Genome Biol 2009, 10(3):R25.

doi:10.1186/1480-9222-16-1

Cite this article as: Lee et al.: Improved reduced representation bisulfite sequencing for epigenomic profiling of clinical samples. Biological

Procedures Online 2014 16:1.

\section{Submit your next manuscript to BioMed Central and take full advantage of:}

- Convenient online submission

- Thorough peer review

- No space constraints or color figure charges

- Immediate publication on acceptance

- Inclusion in PubMed, CAS, Scopus and Google Scholar

- Research which is freely available for redistribution 